\title{
COMPLEX SYMMETRY OF WEIGHTED COMPOSITION OPERATORS ON A HILBERT SPACE OF DIRICHLET SERIES
}

\begin{abstract}
XINGXING YAO
Abstract. In this paper, some characterizations of symbols $\phi$ and $\varphi$ are provided when the weighted composition operators $C_{\phi, \varphi}$ are complex symmetric on the Hilbert space of Dirichlet series with square summable coefficients. As an application, it is proved that there are non-trivial complex symmetric and non-normal weighted composition operators, although this phenomenon happens for neither composition operators nor multiplication operators.
\end{abstract}

Mathematics subject classification (2020): Primary 47B33; Secondary 30B50, 46E15.

Keywords and phrases: Weighted composition operator, Dirichlet series, complex symmetry.

\section{REFERENCES}

[1] M. BAILleul, Composition operators on weighted Bergman spaces of Dirichlet series, J. Math. Anal. Appl., 426 (2015) 340-363.

[2] F. BAYART, Hardy spaces of Dirichlet series and their composition operators, Monatsh. Math., 136 (2002) 203-236.

[3] F. BAYART, Compact composition operators on a Hilbert space of Dirichlet series, Illinois J. Math., 47 (2003) 725-743.

[4] C. COWEn AND B. MACClueR, Composition operators on spaces of analytic functions, CRC Press, Boca Raton, 1995.

[5] C. Finet, H. Queffélec And A. Volberg, Compactness of composition operators on a Hilbert space of Dirichlet series, J. Funct. Anal., 211 (2004) 271-287.

[6] S. GARCIA AND C. HAMMOND, Which weighted composition operators are complex symmetric?, Oper. Theory Adv. Appl., 236 (2014) 171-179.

[7] S. Garcia And M. Putinar, Complex symmetric operators and applications, Trans. Amer. Math. Soc., 358 (2005) 1285-1315.

[8] S. Garcia AND M. Putinar, Complex symmetric operators and applications II, Trans. Amer. Math. Soc., 359 (2007) 3913-3931.

[9] S. Garcia And W. Wogen, Complex symmetric partial isometries, J. Funct. Anal., 257 (2009) 1251-1260.

[10] S. Garcia And W. Wogen, Some new classes of complex symmetric operators, Trans. Amer. Math. Soc., 362 (2010) 6065-6077.

[11] J. Gordon AND H. HedEnMALM, The composition operators on the space of Dirichlet series with square summable coefficients, Michigan Math. J., 46 (1999) 313-329.

[12] H. Hedenmalm, P. Lindqvist and K. Seip, A Hilbert space of Dirichlet series and systems of dilated functions in $L^{2}(0,1)$, Duke Math. J., 86 (1997) 1-36.

[13] S. JUnG, Y. KIM, E. Ko AND J. LEE, Complex symmetric weighted composition operators on $H^{2}(\mathbb{D})$, J. Funct. Anal., 267 (2014) 323-351.

[14] E. Ko, AND J. LeE, On complex symmetric Toeplitz operators, J. Math. Anal. Appl., 434 (2016) $20-34$.

[15] S. NARAYAn, D. Sievewright AND D. Thompson, Complex symmetric composition operators on $H^{2}$, J. Math. Anal. Appl., 443 (2016) 625-630.

[16] S. Narayan, D. Sievewright And M. TJani, Complex symmetric composition operators on weighted Hardy spaces, Proc. Amer. Math. Soc., 148 (2020) 2117-2127. 
[17] S. Noor, On an example of a complex symmetric composition operator on $H^{2}(\mathbb{D})$, J. Funct. Anal., 269 (2015) 1899-1901.

[18] H. QUEFFÉLEC AND K. SEIP, Approximation numbers of composition operators on the $H^{2}$ space of Dirichlet series, J. Funct. Anal., 268 (2015) 1612-1648.

[19] J. Shapiro, Composition operators and classical function theory, Springer, New York, 1993.

[20] M. WANG AND X. YAO, Some properties of composition operators on Hilbert spaces of Dirichlet series, Complex Var. Elliptic Equ., 60 (2015) 992-1004.

[21] M. WANG AND X. YAO, Invariant subspaces of composition operators on a Hilbert space of Dirichlet series, Ann. Funct. Anal., 6 (2015) 179-190.

[22] M. WANG, X. YAO AND F. DENG, Weighted composition operators on the Hilbert space of Dirichlet series, Acta Math. Sci., 38B (2018) 1427-1440.

[23] X. YAo, Complex symmetric composition operators on a Hilbert space of Dirichlet series, J. Math. Anal. Appl., 452 (2017) 1413-1419.

[24] X. YAO, Toeplitzness of composition operators on a Hilbert space of Dirichlet series, Complex Var. Elliptic Equ., 63 (2018) 625-639.

[25] K. ZHU, Operator theory in function spaces, in: Mathematical Surveys and Monographs, vol. 138, Amer. Math. Soc, Providence, 2007. 